faithful guardian of the natural world. Mention of evolution is now banned too, as presumably too controversial, and this in an institution that seeks increasingly not only to encourage school parties but also to provide biological instruction to schools on a vast scale through television and the Internet. There are important issues here, on which Davis has shone a remarkable light. Even to persuade such an institution to let her interview its staff - without editorial control is no mean achievement.

Vicki Croke's account of the history of zoos in The Modern Ark is a less original study and on an altogether smaller scale. But it is superbly written, with an enviable lightness of touch, which is just right for expressing the magic of the best in zoos and the opportunities they sometimes offer for real appreciation of their animals, such as gorillas at Chicago's Lincoln Park or a fur-seal watched (and watching back) through an underwater viewing panel at Tacoma, Washington. Some of Sea World's exhibits, such as a splendid Antarctic simulation for penguins, must also belong in this category (apart, for me, from the music). Croke's gorilla and seal examples come near the start of her book, partly as a backcloth for her recognition of zoos' all too frequent failure to live up to their own aspirations.

Hers is thus a fair, even-handed account. And it is a well informed one, especially regarding new developments in zoos, in the United States in particular, and her account of the earlier history of zoos is refreshingly lively. Particularly useful are her wise discussions of such controversies as the pros and cons of keeping white tigers (or even white lions) and the appalling problem of whether zoos are justified, even for high conservational motives, in culling so-called surplus animals.

Stephen Bostock is at Glasgow Zoo, Uddingston, Glasgow G71 7RZ, UK.

\section{Money spinner}

\section{Fractals and Scaling in Finance}

\section{by Benoit B. Mandelbrot}

Springer: 1997. Pp. 456. \$39.95, £32.50

\section{lanStewart}

In the late 1950s and early 1960s, Benoit Mandelbrot, then a young and relatively unknown researcher at IBM's T. J. Watson Research Center, devoted a lot of his time to problems in economics and finance. His ideas in these areas formed a tiny part of a huge body of work, ranging from rainfall statistics to linguistics, that led him to create the concept of the fractal, for which he is now famous all over the world.

Fractals and Scaling in Finance brings Mandelbrot's work full circle, applying today's mature fractal geometry to the problems that plagued him 40 years ago. It is a typically Mandelbrotian mix of reprinted papers, commentary, unpublished results and new work hot off the press. Its focus is simple: what is the structure - if any - of financial data? Its scope is so broad that I cannot hope to summarize it adequately.

The world's stockmarkets have recently been in turmoil as the FTSE 100 Index and the Dow Jones plunged headlong after the Nikkei and the Hang Seng. Some weeks the market has a clear dynamic, and it is down; but sometimes it is up. Who knows which it will be next week? Faced with such unpredictability, classical mathematics took one look at the financial world, deemed it to be random, and the paradigm was set. The great triumph of modern financial mathematics - the famous Black-Scholes equation of 1973, without which there would be no derivatives market - is based on a purely stochastic model.

Just as biologists 'know' that evolution is random - despite a wealth of evidence to the contrary - so financial analysts 'know' that the market is random. The biologists' mistake is to confuse a dynamic with a goal. Evolution may well have no predetermined goals, but it manifestly has an emergent dynamic. Emergent phenomena are easily confused with random ones, because neither has an accessible 'audit trail' of cause and effect. In random phenomena - or more accurately, in models of them - there is no audit trail, whereas in an emergent phenomenon it exists but is too complicated to describe.

Financial analysts have made the same mistake. The market has all the characteristics of an emergent system, and the evidence that market data possess hidden structure is becoming overwhelming. Measures of the degree of order in a time series, such as the 'approximate entropy' introduced by Steve Pincus, reveal that the markets generally remain well below the maximum level of disorder. The only time the Dow Jones index came close to maximum disorder was immediately before the stockmarket crash of 1987.

But what kind of structure do market data possess? According to Mandelbrot, their central feature is scaling. Roughly speaking, the small-scale fluctuations of the market mimic the large-scale ones, but on a compressed timescale. The genesis of this principle was Mandelbrot's early realization that orthodox statistics can tell us very little about financial data. The reason, he believed, is that for financial data the standard deviation is infinite. This implies, for example, that jumps in market value can be bigger, and more rapid, than conventional statistics would allow.

The simplest statistical distributions with this feature are power-law distributions, a far cry from the beloved (and dreadfully overrated) Gaussian of the textbooks. In a way, the entire book is the story of Mandelbrot's intellectual voyage into power-law territory, as he refined his early observations into more sophisticated, but always simple and elegant, models of the irregularities of financial time series.

The high point is the theory of L-stable processes and their generalizations, which capture many characteristics of market data in both qualitative and quantitative ways. It would be interesting to relate these ideas to recent work that models markets as 'adaptive complex systems', which are collections of entities (traders) interacting according to simple rules, from which the overall market emerges. There is growing evidence that such systems capture many features of financial markets, and may even be a way of finding that elusive element of 'structure'. Are there credible micro-rules that generate macro L-stable statistics?

This is not an easy book to read, but once one gets into the flow of ideas it is rewarding and full of insight, and should be on every chartist's bookshelf. There is one weakness, though, that will be apparent to any child of the computer age: a neglect of modern data. Mandelbrot explains that, although extensive data are now available, he is "ill equipped for empirical work". Perhaps... but isn't that what postdocs are for?

Ian Stewart is in the Department of

Mathematics, University of Warwick, Coventry CV4 7AL, UK.

\section{ADVERTISEMENT}

"Beside good science, there is much humour and much of interest outside the field of science. ... In my case, it kept me awake and delighted until the small hours"

Keith J. Laidler, Professor Emeritus of Chemistry, Ottawa University

\section{The Bedside Book Genius and Eccentricity in Science 1869-1953}

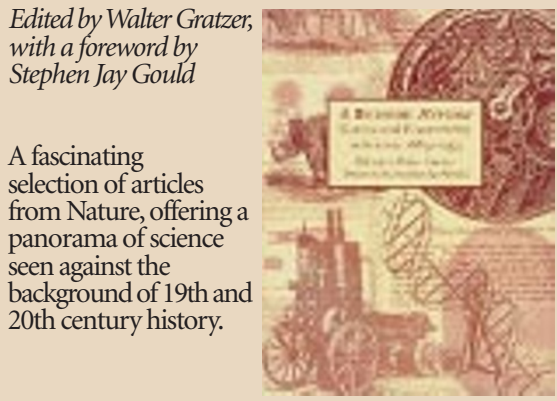

Price: $\mathfrak{£} 19.95 /$ US $\$ 29.95$ + postage

Discount rate available for current subscribers. To order, or for more information, contact: Nature Marketing Tel. +44 (0) 1718434985

Fax. +44 (0) 1718434998

E-mail: subscriptions@nature.com 